\title{
How Constructional Networks Grow and Fade
}

Welcome back to this fifth lecture of Ten Lectures on Diachronic Construction Grammar. In the last lecture, I have started to discuss a number of ways in which constructionalization and constructional change can be studied empirically on the basis of diachronic corpus data. In this lecture, I will continue with that general theme. The title for this lecture is "How constructional networks grow and fade". The studies that I presented in the last lecture elaborated on the idea that constructional meaning is reflected in patterns of associations between syntactic structures and lexical elements. I explored the idea of a constructional network, in which grammatical constructions entertain many connections with lexical elements that occur with these constructions. A construction like the English auxiliary will, for example, has a slot for a verb in the infinitive. That slot is connected via hundreds and hundreds of associative links to different lexical verbs that can fill that slot. Importantly, these links have different strengths. Associations differ in how strong they are, also the strengths of these connections can change over time. You can examine how a given construction is connected to lexical elements in the 1800 s and how that changes over the years, so that you have a very different situation in the 20oos. Collostructional analysis, the method that I have used there, allows you to investigate that.

The overall phenomenon that I have addressed is connectivity change. This is what you see displayed schematically on the slide. The examples that I have discussed were future constructions in Danish, Dutch and English that illustrate the general point. This idea of connectivity change and how you can study it was the general theme of my 2008 book. If you would like to go deeper into that issue, you can turn to the book, which presents a range of similar studies that explore that idea from different angles. In this lecture I want to put the focus on another of the ten basic ideas of Construction Grammar that I presented to you earlier. Namely, I want to focus on the idea that constructions vary in terms

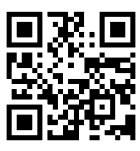

All original audio-recordings and other supplementary material, such as any hand-outs and powerpoint presentations for the lecture series, have been made available online and are referenced via unique Dor numbers on the website www.figshare.com. They may be accessed via this QR code and the following dynamic link: https://doi.org/10.6o84/mg.figshare.13691107. 
of their degrees of complexity and schematicity, which has profound implications for the way we think about constructional networks. I will talk about a perspective on changing constructional networks that I developed in my 2013 book on constructional change. Constructional change, if you remember the definition from Lecture 2, selectively seizes a conventionalized form-meaning pair. It changes a construction in terms of its form, its frequency, aspects of meaning, and its distribution in the community who uses it and any combination of these factors. The networks of changing collocational associations that I described in the last lecture mainly focused on two aspects of this definition, namely changes in frequency. We saw that different collocates become more frequent or less frequent, and this is picked up by the collostructional analysis. The second aspect is that these future constructions also change in function. As a future construction moves along its grammaticalization path, it becomes broader in its meaning potential. Its function changes. Crucially, the constructions that I described yesterday did not undergo any formal morphosyntactic changes during the historical period that I investigated. I was really exploring what Traugott and Trousdale would call constructional change, rather than constructionalization.

Today I would like to present a study of constructional change that does involve formal change alongside functional change and frequency change. I will also focus on a different grammatical domain. Yesterday, we were firmly in the domain of verbal grammar, studying the behavior of verbs and auxiliaries. Today, we turn to the nominal domain. I will be talking about English nouns, and specifically about morphology and word formation, discussing how new words enter the language.

The case study that I brought along for this morning concerns an English derivational suffix that has had an interesting life and death. The suffix by itself is -ment, which is a nominalizing suffix that you know from English words such as punishment, treatment, settlement, and many others. I became interested in that particular suffix, because I noticed something strange about it. In Present-day English, you find around 1000 words ending in the suffix -ment. Dictionaries list many different words of this kind. If we investigate -ment on the basis of corpus data, we find that many of these noun types are relatively infrequent or occur only once. A word that occurs only once in a corpus is what corpus linguists call a "hapax legomenon". Morphological patterns with lots of unique types, lots of hapax legomena, are typically very productive, because the large number of low-frequency types shows that speakers use that suffix to create new words spontaneously. That is normally the case. But with -ment, even though there are lots of different types and lots of infrequent types, speakers cannot produce new words on the basis of -ment. Let's say that I have been 
emailing back and forth with a colleague this morning. I could describe this as a long emailment. That, however, is not a possible word of English. People would understand it if I say it. But they would also understand that I am making a language-based joke. I would be doing something that is not within the limits of what I can conventionally do with the suffix-ment. The same would be true of recyclement. I can collect my glass bottles and my old newspapers and then show them to a friend and when I say, "These here are my recyclements." My friend would understand me, but it would not make recyclement a usable word. I was wondering, how can I explain this discrepancy? What is going on with the suffix -ment?

In the time that I have this morning I want to focus on four questions. First of all, what is the $V$-ment construction? I call it the $V$-ment construction, because the stems that you find in the construction are typically verbal. A form like punishment has the verb punish and -ment makes a noun out of it. The word treatment starts with the verb treat, and -ment makes a noun out of it. How can we describe that construction?

Second, how did the construction change in productivity? It must have been productive at some point. At some point, speakers of English produced new types on the basis of that suffix, but somehow that stopped.

I also want to explore how this construction changed over time in form and function. What are the different meanings that can be described with it? What are the different forms that we find in it? For example, even though I just said that the construction normally occurs with verbs, as in treatment or punishment, there are some forms that are exceptions to that. If you think of something like basement, we have base plus -ment. Base is arguably not a verb in this context, although it exists as a verb elsewhere.

Lastly I want to bring it all back to Construction Grammar and the question of constructionalization and constructional change. How do these findings about productivity and change in form and function speak to the issue of constructional change?

Let's start with a general characterization of the construction. I have already mentioned that it is a combination of a lexical stem with a suffix that has a certain phonemic shape. The suffix is pronounced as -ment, and the stem strongly tends to be verbal. I have mentioned the exception of pavement already. There are others, for instance, merriment, which is an infrequent, somewhat archaic word that describes joyful activities. Meaning-wise, the construction typically conveys the meaning of an action. An adjustment is the action of adjusting a projector that is sitting a little bit askew. It can also be the result of an action. When I buy an assortment of sweets, an assortment is not the action of putting the sweets in the box, but rather it is the box that I buy and I can take 
home. The construction can also express the means to accomplish an action. A refreshment is not the action of refreshing myself by taking a sip of water, but rather it is the water that I can drink or small snacks that I can eat.

Let us move on to what happened to -ment historically. This slide shows a very short summary of its history. It starts in Old English, where we can already see a few words that end in -ment, and those were loan words from Latin. Old English had borrowed and incorporated words from Latin that were part of ordinary language use. But those were isolated forms. The construction as a generalization came about as a contact phenomenon, due to an important event in the history of the English language, namely, the Norman Conquest of 1066. The -ment suffix is of Romance origin, and that is how it entered into English. The construction then became nativized. We see that in forms with -ment that do not have French verbal stems. Take a word with a Germanic stem like shipment for instance. Ship is a Germanic word, and we find it combined with -ment. We find the Romance construction with Germanic parts in it. That is how we can know that this construction was actually incorporated and nativized into English. That happened between 1250 and 135 .

Shortly after that, the construction already receded and became less productive and fewer and fewer loan words were borrowed into English. That already marks the downfall of the construction. The overall productivity receded, and what we have in Present-day English is what you could describe as a residue, leftovers of history. About thousand types remain in the language, but the construction is not productive. I have given you the examples of emailment and recyclement earlier. Today I have been going for a run that I cannot describe as a jogment, or if I am kissing someone repeatedly, that cannot be described as a kissment.

How did the construction change in productivity? How can that be analyzed? I decided to use a database for the study that I talked about yesterday in the context of Michael Israel and the way-construction. Michael Israel used the Oxford English Dictionary for his study, and I did the same for my investigation into -ment. The Oxford English Dictionary is not a diachronic corpus. At least it is a very special type of diachronic corpus. It is a dictionary that has words and definitions, but above and beyond that, it has authentic examples of texts. It has quotations. These quotations have historical dates.

Let me show you a screenshot of what the electronic version of this dictionary looks like. This slide shows the entry for the word achievement. You see that there is a section on the etymology of achievement. It comes from Anglo-Norman and Middle French. It means the action of finishing or completing something. We are given information about the general time during which that word was borrowed. Below that are authentic examples that the compilers 


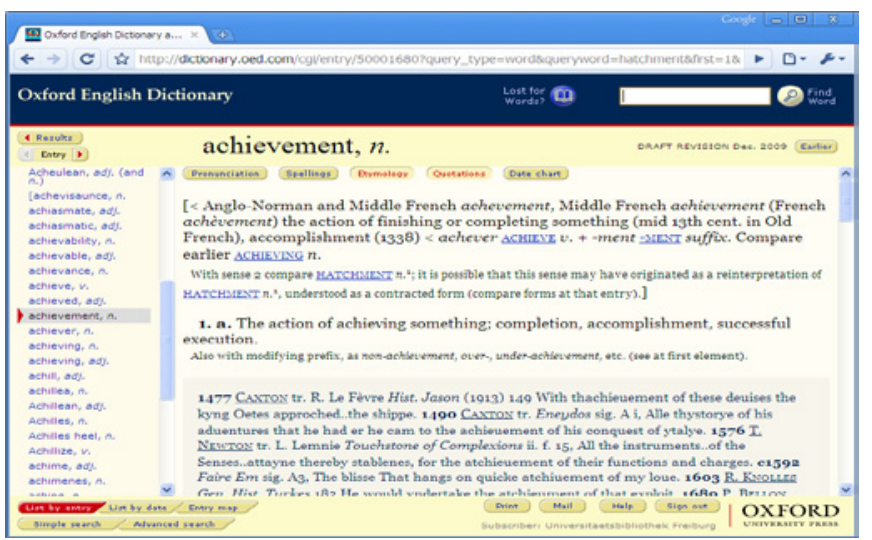

FIGURE 1

of the Oxford English Dictionary have collected. That allowed me to take the first date of attestation as a proxy for the time when this word would have entered the English language. Much like Michael Israel was collecting verbs with the way construction, I have been collecting -ment words from the Oxford English Dictionary, taking notes as to when these words were first attested and when they first entered the language. That allowed me to track over time how many new words with -ment entered the language at any given point in time.

\section{Data from the OED ( 1400 types)}

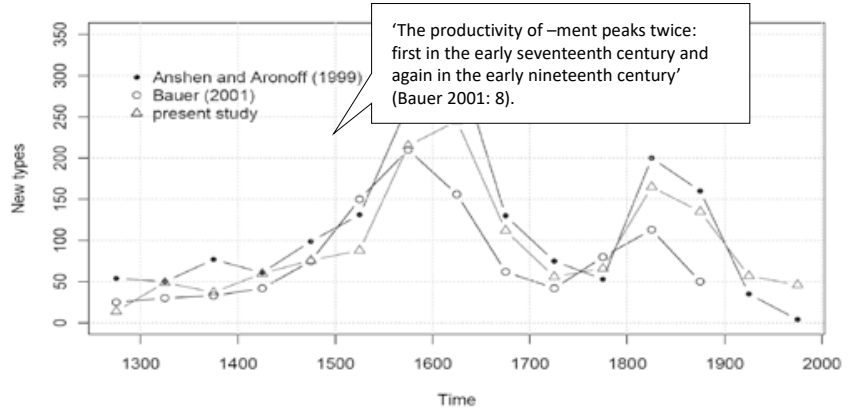

FIGURE 2 
Proceeding in this way, I found about 1400 different words ending in -ment that were recorded in the dictionary. I did this and as soon as I was finished, I realized that there had been two other studies that had been doing the exact same thing (Anshen and Aronoff 1999, Bauer 2001). I was very nervous to see how their data would compare to mine. You can see in this graph on the slide three frequency curves that show how many new types with -ment enter the English language during every half century. What you see is that the three curves are in broad agreement. At least they are not very far away from each other. There are a few discrepancies here and there. For instance, Anshen and Aronoff (1999) find more types around the year 160o. In the very last period, I found more types. That is because the Oxford English Dictionary is continuously updated with new entries. Between 1999 and my study in 2013, there were new types that were added, and that accounts for this difference.

When you look at this curve, you notice that it looks like the back of a camel. Bauer (2001) as argued on the basis of this curve that the productivity of -ment has two peaks. It has two periods at which it is a very productive, first in the early 17 th century, and then again in the early 19th century. Now, that statement is problematic because the texts that were compiled by the editors of the Oxford English Dictionary are not of the same size across all historical periods.

Quite to the contrary, later periods are represented with lots and lots more text. That means that at the end of the period we are much more likely to find lots of words that end in -ment. What I decided to do as a first analytical step was to normalize the type frequencies. By calculating the number of types per 10,0oo words I tried to control for the different amounts of text that we find for each historical period.

\section{Normalized type frequencies}

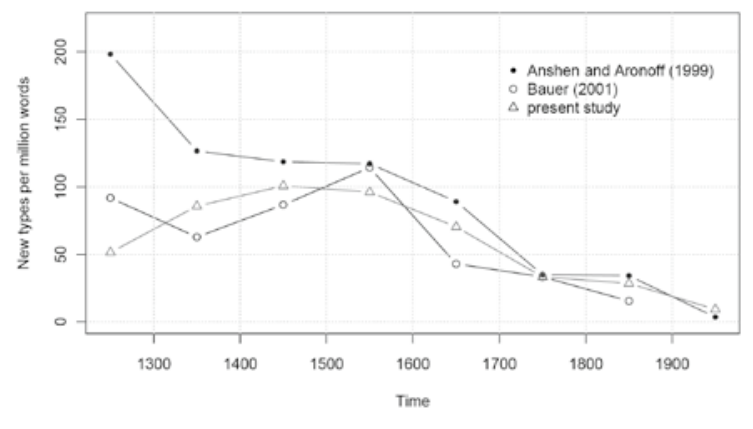

FIGURE 3 
Once I did that, the camel shape disappeared. What we see here are the normalized type frequencies. In the beginning, there are some discrepancies, about up to $155^{\circ}$. But after that, all the three curves seem to agree that there is a linear, gradual decrease of new types per time period. That means that from the middle of the 16th century, this construction has been decreasing in productivity more or less steadily. Normalized type frequencies are one way of approximating the concept of productivity, how easily new types are formed, but it is not a very precise measure. It is not a measure that is typically used in current corpus linguistic studies of productivity. Typically, corpus linguistic studies assess productivity through measures that take into account the prevalence of hapax legomena.

\section{Expanding productivity}
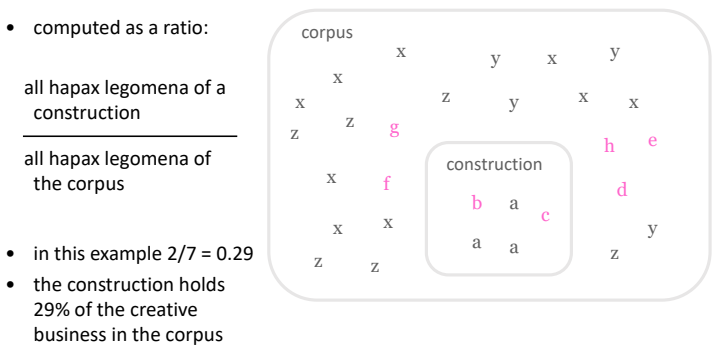

FIGURE 4

I decided to apply a corpus linguistic measure that is labeled expanding productivity. This measure of productivity is calculated as a ratio of hapax legomena in a construction and hapax legomena in the corpus. You see a visualization of the general logic of this measure on this slide. Again, we have a corpus with lots of different words in it. The words are represented by letters like $x, y$ and $z$. You also see an $f$ and a $g$.

I am interested in the productivity of this construction here. The first analytical step is to extract all the types of that construction. In this toy example, we only have three different types, $a, b$ and $c$. Two of them occur only once. There is only one $b$, there is only one $c$, so they are counted as hapax legomena. The three instances of $a$ occur more than once, so they are not counted.

The number of all hapax legomena in the construction has to be divided by all hapax legomena that I find in the entire corpus. In the entire corpus, there are lots of $x$ s. They do not count. There are lots of $y$ s and $z$ s that do not count 
either. By contrast, there is only one $f$, only one $g$, only one $h$, one $e$, one $d$, one $b$ and one $c$.

Following this logic, we would divide 2 by 7 . There are 2 construction hapaxes, and there are 7 corpus hapaxes. That would give us the ratio of all hapaxes that are accounted for by the construction. It gives us the part of the creative language use that the construction represents in the corpus. We could say that the construction accounts for $29 \%$ of the creative language use in the corpus. I should maybe say why hapaxes are taken as a representation of linguistic creativity. When I am doing something creative with language, when I am making up a new word, then that word will at first be only very infrequent. The first time I say a new word, it occurs only once. Speakers are creative more or less all the time. They bring new words into their languages all the time. At first, these words are very infrequent. Corpus linguists look to infrequent word types to make inferences about how speakers are creative with their languages.

I calculated this measure of expanding productivity for all time slices in the OED (Oxford English Dictionary) that I had. For each time slice, I counted the -ment hapaxes and the overall number of hapaxes in the OED quotations for that time slice. Of course, not all hapaxes are words that are created in that very moment. Some words are just very infrequent, but for those words that are creative, we can be relatively sure that at first they are likely to be used with low frequency.

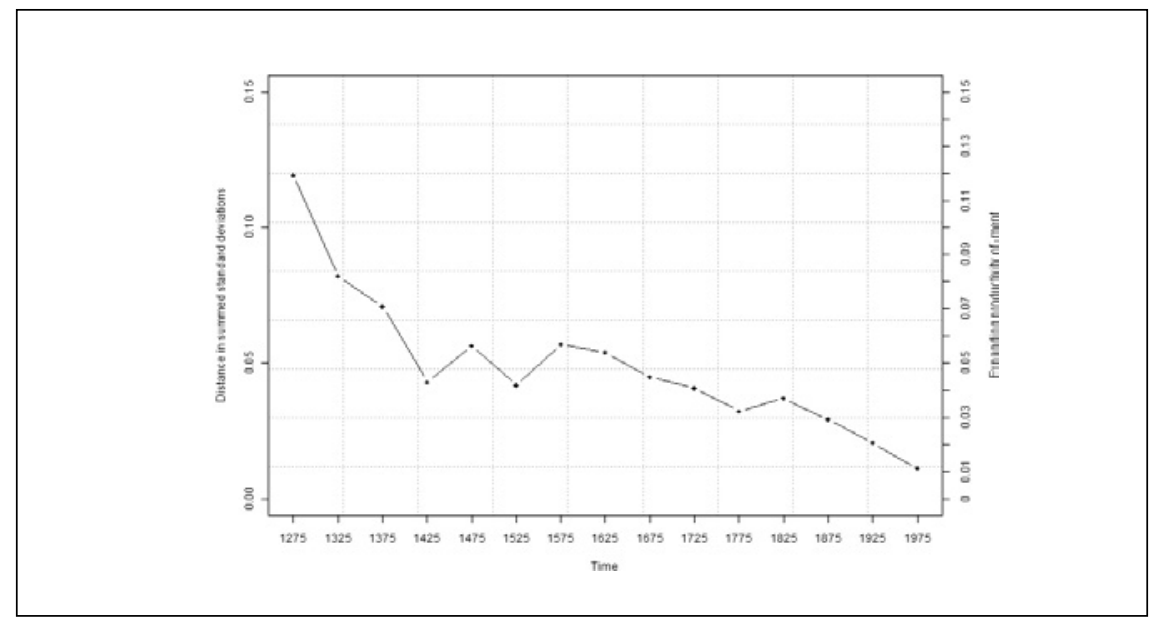

FIGURE 5

This calculation of expanding productivity over time gives me the descending curve that you see on this slide, which looks very similar to the curve of normalized type frequencies that I presented earlier. This suggests that this almost 
uniform downward curve represents the decline of productivity that the -ment construction undergoes.

To sum this up, how did the V-ment construction change in productivity? My conclusion was that there is really just one peak in productivity, and that peak occurs relatively early during the 13th century, which marks the moment of nativization. This was borrowed into the English language and it was nativized. It was popular for a while, but then its popularity faded quickly. I tried to find an non-linguistic analogy for this. You probably know the Rubik's cube, which is a plastic cube with multi-colored squares that you can twist and turn in order to make each side appear in just one color. It was a very popular children's toy at some point in time, everybody needed to have one. The analogy actually goes further than a toy being highly popular at one point and then falling out of fashion. If you go through a box of old toys in your basement, you might still find a Rubik's cube. In similar ways, you still find all the old -ment nouns in the English language. They're still there. We still use them. We just do not make new ones.

Moving on to the next question, how did the $V$-ment construction change in form and function across its lifespan?

In order to analyze this, I decided that I wanted to divide the history of the construction into stages, so that I could find out what happens early on and compare that what happens in the middle of the development and at the end. I thus needed a way to determine how I wanted to divide the overall development into stages. In historical linguistics, this is a general question. How do we divide a stretch of time into periods? Do we take one century, then the next century, then the next century? Do we take decades, or even individual years? Or do we go with periods that are motivated on the basis of language-external events? If there are important historical events that affect the language and the culture, that might lead us to distinguish time periods based on those events.

Stefan Gries and I had been developing a method that we called variabilitybased neighbor clustering or vNC. The general idea of that technique is to arrive at a periodization of historical data that is inductive and data-driven. If the data does not change significantly during one particular time, that time should be recognized as a coherent period. If the data changes suddenly, then we have a reason to posit a boundary between historical periods. Variability-based neighbor clustering is a clustering algorithm that adopts the general principles of other hierarchical clustering methods. Unlike other clustering algorithms however, there is one twist in neighbor clustering, namely that it can only merge temporally adjacent data points. I will explain what this means in just a second. 
Three stages?

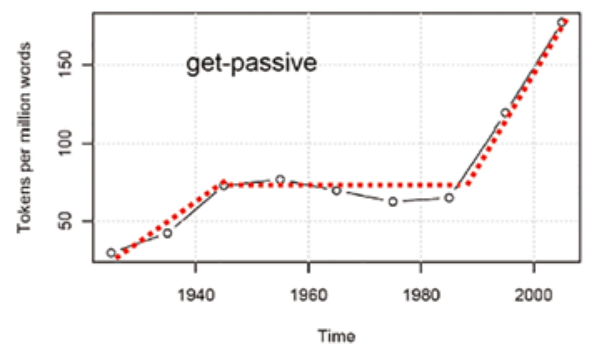

FIGURE 6

In order to explain it, I need to leave the V-ment construction for a moment. Let us look at one or two simpler examples. Here we see a frequency curve for the English get-passive construction which shows a diachronic increase. This is a data from The тіме Corpus of American English. You see that it starts low, then there is an increase, then there is something of a plateau. After 1980, the construction strongly increases in frequency. How do we partition a frequency curve like this into stages? You could look at this curve, eyeball it, and divide it into an initial period of increase, a second period with a plateau, and a third period with a strong increase.

When we partition diachronic corpus data into periods, there are several potential pitfalls. Typically, diachronic corpus work divides data into sequential periods that are chosen arbitrarily, like centuries or half-centuries or decades. But problem with that is that linguistic change is not always smooth. It can move in fits and bumps, and there can be U-shaped curves. That means that when we measure a linguistic phenomenon over a certain period and take an average value, the results that we get may actually be misleading in some cases. Different time slices, different periodizations yield potentially different results. The ideal way to divide the corpus into time slices would be to take some aspect of the phenomenon that is studied and to have a data-driven way of periodizing the data. How can that problem be addressed? One way of finding structures in large bodies of data is hierarchical clustering.

I think that at least some of you are familiar with the general idea of the method and its applications across different scientific disciplines. Clustering is used, for example, in biology, where researchers use it to investigate similarities 
in the DNA of different beetle species. The method allows us to get a sense of how groups of species form larger groups. Clustering is of course also applied in linguistics.

My former officemate, Benedikt Szmrecsanyi, has used clustering to find groups of English dialects on the basis of their morphosyntactic characteristics (Szmrecsanyi 2013). Benedikt didn't measure DNA sequences, but instead took a catalogue of more than 50 morphosyntactic features, and he measured how often these features occurred in corpus data that represents these dialects. Let me say a few words about how this works in principle.

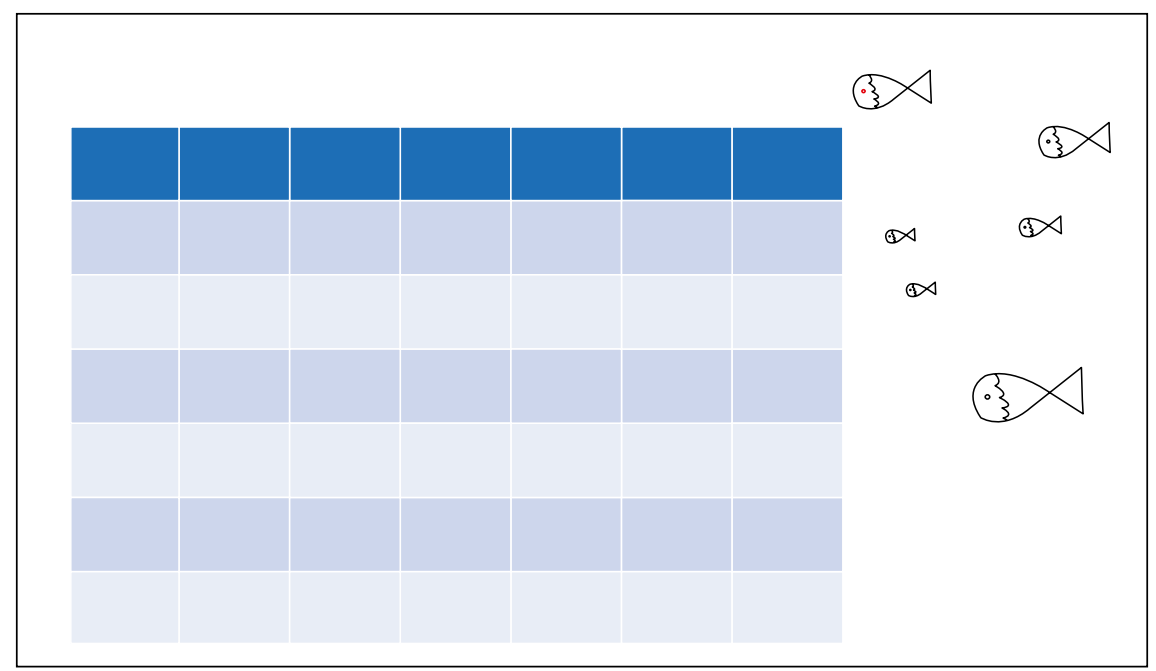

FIGURE 7

We'll take a very simplistic example, with six elements that differ in just one feature, namely physical size. There are six fish that differ in how large they are. How do we divide these six into groups? It would be possible to describe the set as one big fish, two medium-sized fish, and three small fish. It would also be possible to distinguish between three large ones and three small ones. Hierarchical clustering offers an inductive way of deciding how to describe the set. We arrange the fish in the matrix like this. Each fish is compared to each other fish.

We determine the size difference between one fish and all of the others. Comparing the big fish to itself yields a difference of o. A comparison with the next one yields a difference of 5 . The next one shows a difference of 9 , and so on and so forth. For every possible pairing, we determine a measurement of difference. The difference between the two smallest ones is 0.5 , which is circled on this slide. When all difference measurements are taken into account, this is the smallest difference between all combinations in the entire set. 


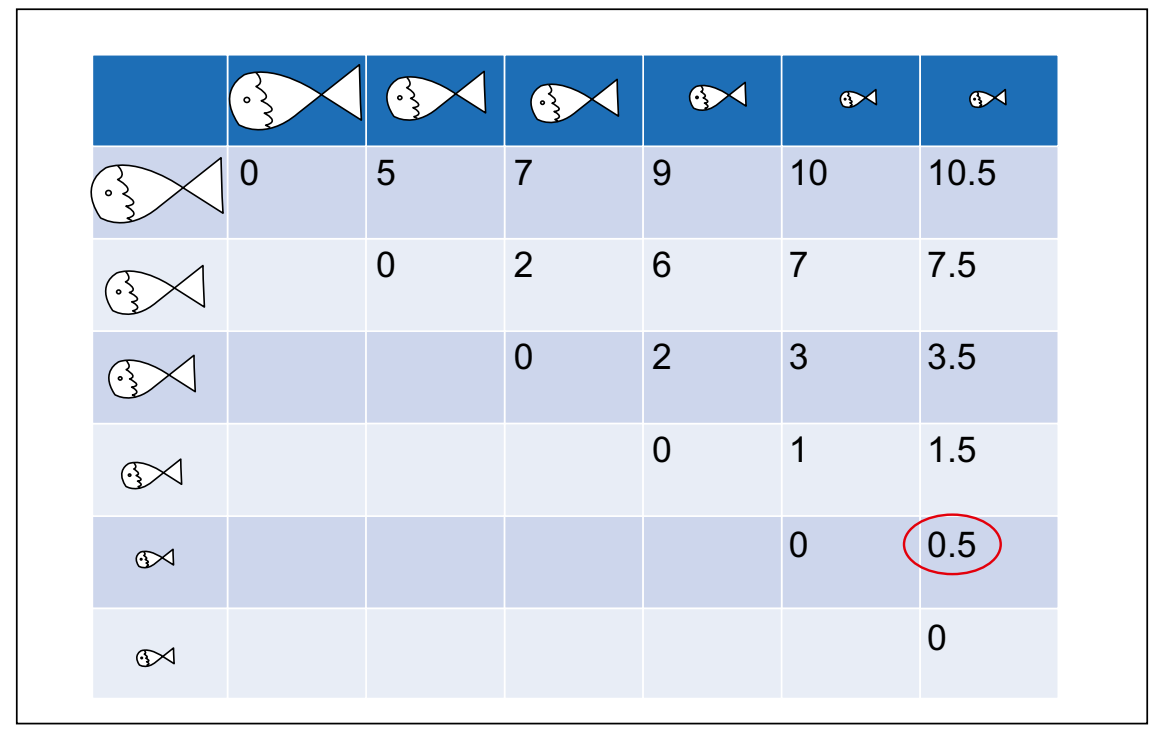

FIGURE 8

The starting point of a clustering algorithm is the establishment of differences on the basis of pairwise measurements of all the data points that you give it. The algorithm determines a measure of difference and looks for the smallest difference in the entire dataset. Once this pairing has been identified, once we have the two fish that are the least different, the algorithm puts these together and calculates an average value, so that we have a table that is a little bit smaller.

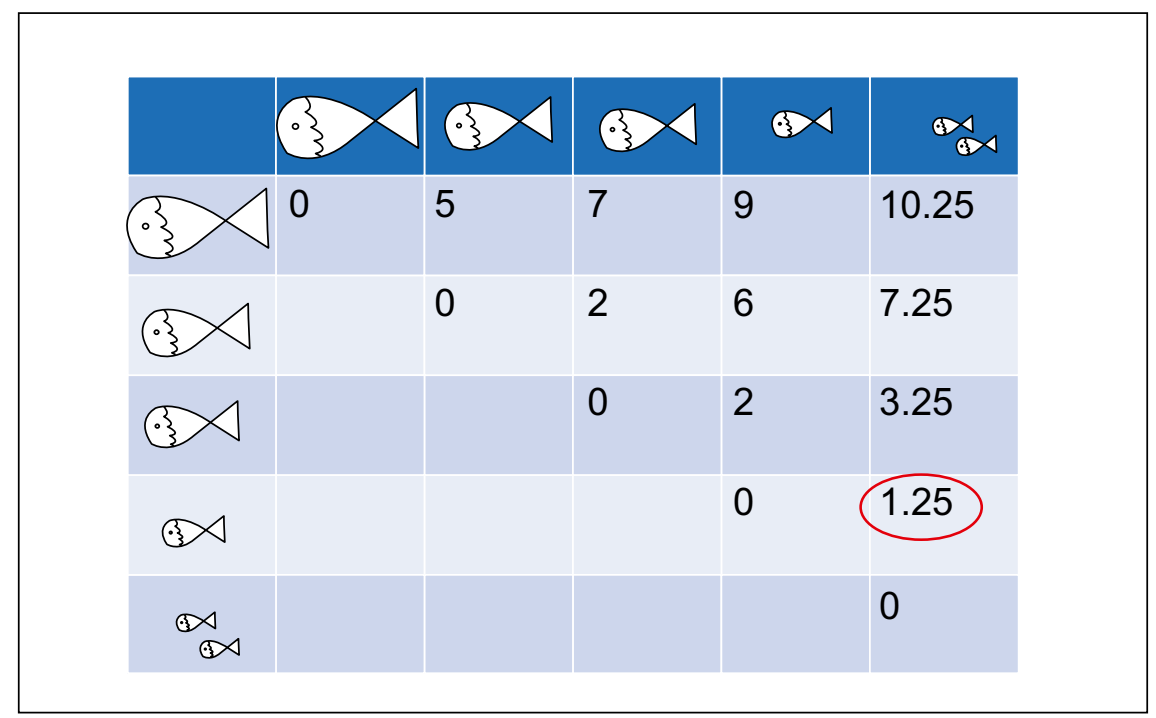

FIGURE 9 


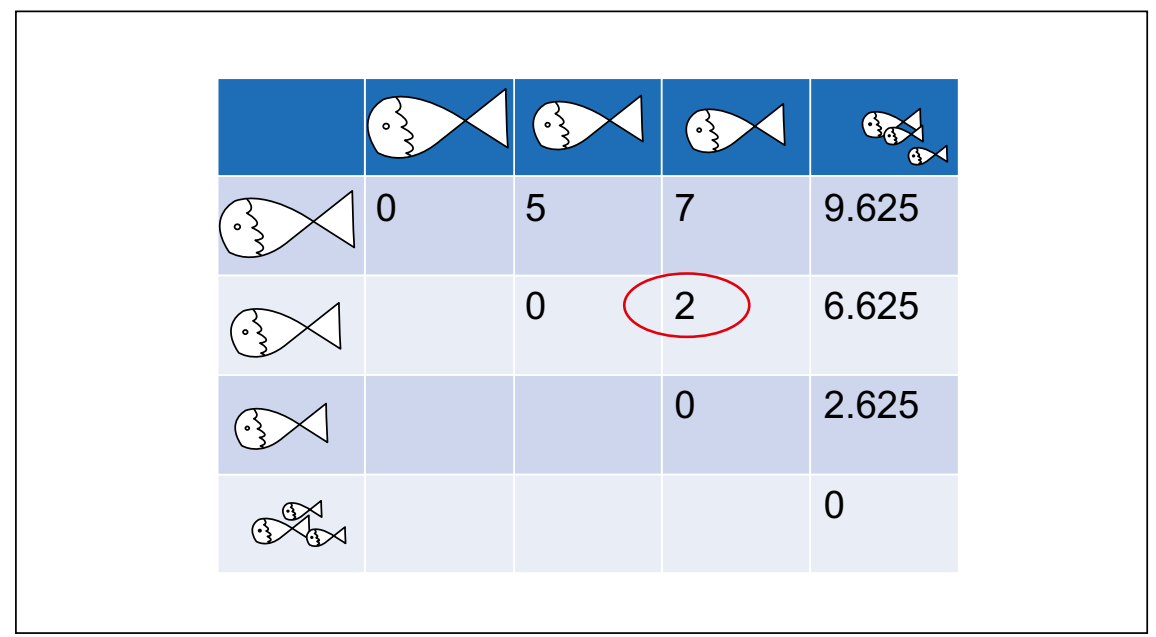

FIGURE 10

Now we have a matrix in which these two have been merged. They form a cluster. The average size is taken to compute a difference between the biggest fish and this cluster, which is 10.25. With the cluster of the two small fish, we now have a slightly smaller table in which some values are recalculated in the second round of the iterative clustering algorithm. The algorithm takes all these numbers and searches for the smallest difference. In this iteration, the smallest difference is between cluster with the two smallest fish and the smallest remaining single fish.

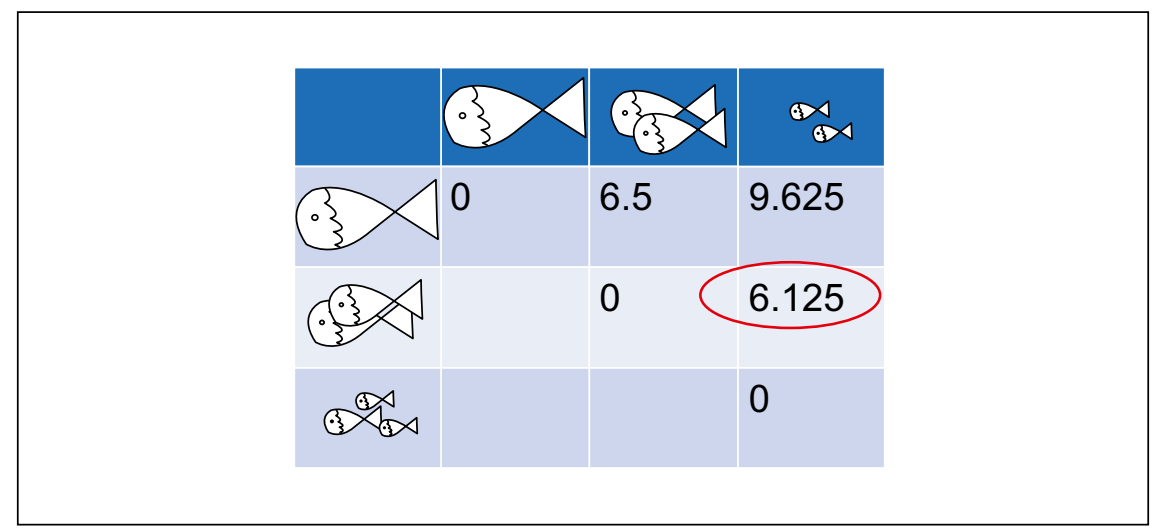

FIGURE 11

In the following iteration, we take the average of that and are left with a table that is even smaller. The algorithm creates the cluster with what is now three 


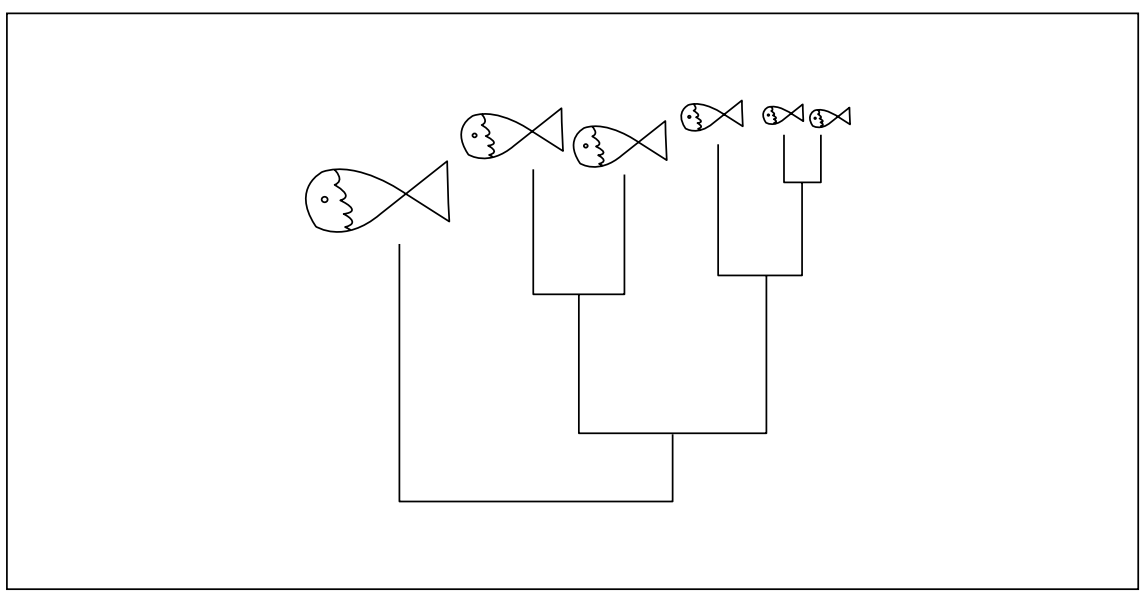

FIGURE 12

fish, recalculates the differences, finds the smallest one, and then the process repeats. This time, the smallest number that we find in this table is the difference between the two medium sized fish.

That means we put these two together and get the smaller table that looks like this. This actually gives you the answer to the question that I asked earlier. Our set contains one big fish, two medium-sized fish and three small fish. The algorithm does not stop there. You can go on with it and the next iteration would group all the smaller fish together.

Overall, we have a clustering dendrogram that looks like this. A useful way to describe this would be one big fish, two medium-sized, and three small ones. But if you wanted to have only two groups, you would differentiate between one a big fish and five small ones.

To return to language, the general idea is that we can use clustering to find how the development of a given linguistic unit can be divided into stages, so that instead of taking fish and measuring their size, we take data from different historical periods and annotate the data for a feature that we are interested in. We could take the frequency of an element. We could take the relative frequency of dislike with the to-infinitive and dislike with the -ing form, or we could take the range of collocates, like Michael Israel did with the wayconstruction. Anything that you can count, anything that you can measure and express in a number, you can study in this way. Then, you take those measurements and group them according to their similarity. General hierarchical clustering can be applied to diachronic data in this way. But there is one problem, namely, conventional hierarchical clustering algorithms do not know about the temporal sequence that you have in your data. 
If, for instance, we have data that comprises data from 1993, 1994, 1995, 1996, and so on and so forth to 2000, it may happen that the data from 1993 is very similar to the data from the 2000. Conventional hierarchical clustering would group the two years together, meaning that you would end up with clusters that are nonsensical. How do we fix that? That is the problem that variabilitybased neighbor clustering addresses. The clustering algorithm measures the differences from one period to the next. We only merge those pairings that are temporally adjacent. We can only combine data points that sit next to each other in their temporal sequence.

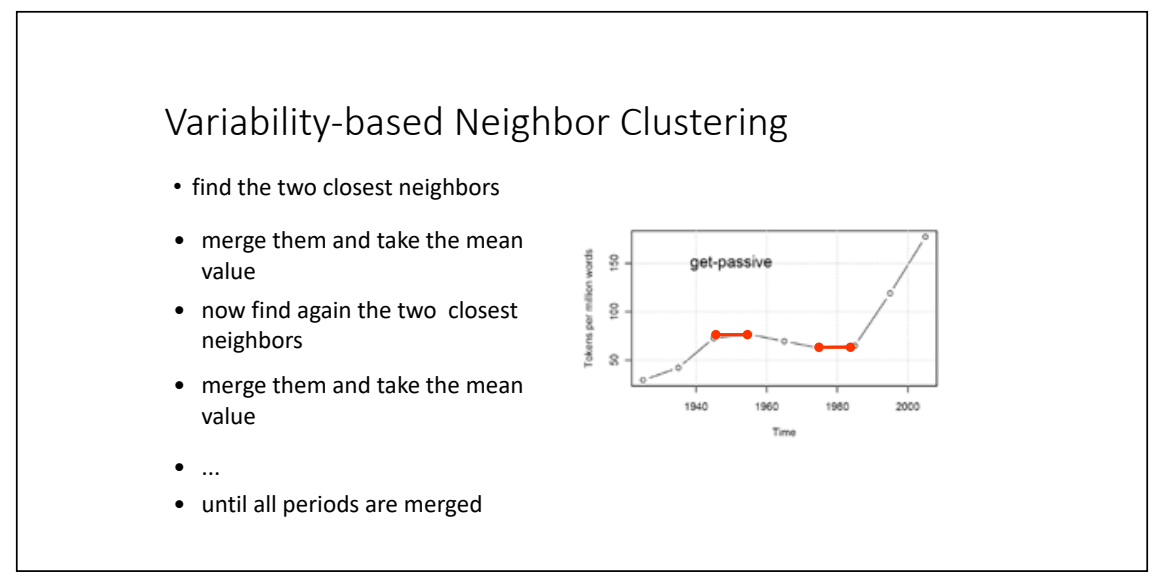

FIGURE 13

Let me come back to the get-passive and explain how this works in practice. The clustering algorithm goes through these data points. For each pairing, it calculates a measure of difference. It goes from the first to the second and measures how different they are. It goes from the second to the third and measures the difference. It goes from the third to the fourth and finds a very small difference. Fourth to the fifth, that is also pretty small. Fifth to the sixth, it is kind of small. Sixth to the seventh, this one is really small. Then the last two ones are quite large again. It finds the closest neighbors, which in this case are the sixth and the seven point here. It merges them. It takes the average value, just as with the fish. Then it goes through the entire set again and finds the two closest neighbors. In this case, on the second iteration of the algorithm, the two closest neighbors are the third and the fourth point. It merges them, it takes the mean value, and it does that again and again until all periods are merged together. Clusters of clusters of clusters until you have everything in one tree diagram. 


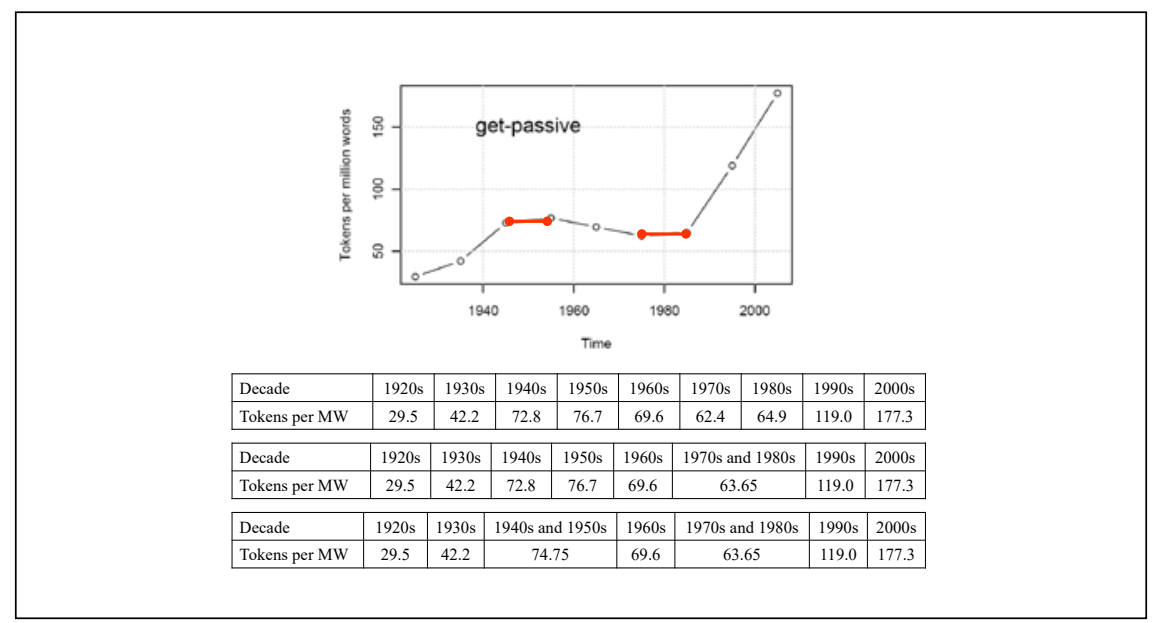

FIGURE 14

Just to go over this with concrete numbers once, we start with all data points, and we find the adjacent pair that is the closest match, which in this case are the 1970 s and 1980s. Those two are then merged. We take the average. This process repeats so that the next time around, the closest neighbors are the 1940s and 1950s. We take the average and the algorithm goes through the data again and again, until all of these cells are merged into one. We have the overall average, which then gives you a tree diagram that reflects the overall development.

In this case, we learn that in order to divide the development into two parts, we would need to distinguish the last one from everything else. If we divide the development into three parts, distinguish the last one, then the penultimate period, and then all the rest. For four parts, we would have the first two periods,

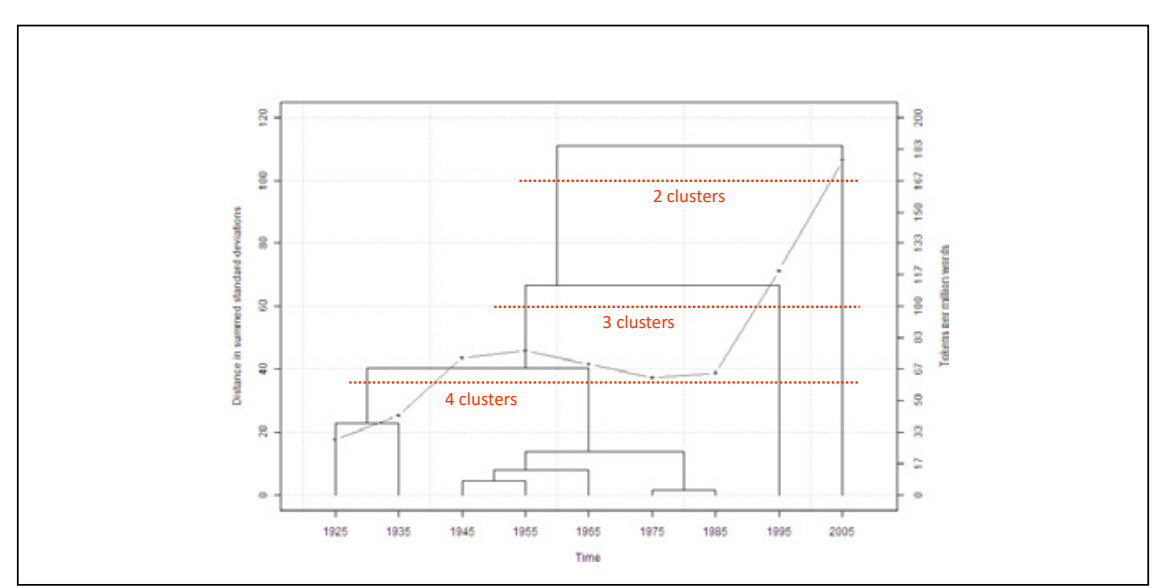

FIGURE 15 


\section{How many clusters?}

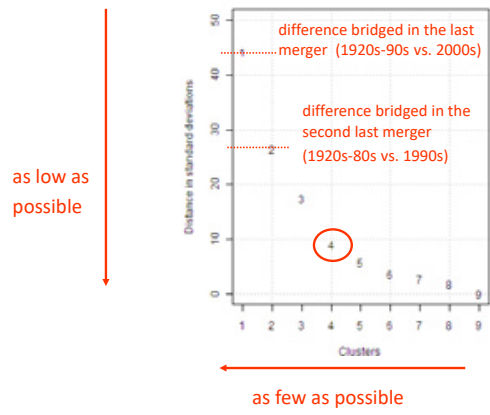

FIGURE 16

then a cluster of five periods that represents the plateau in between, and then the penultimate data point on its own and the last data point on its own. That is how it works. The question is, how many clusters should we assume?

There is no exact science to answer that question. But the difference between each merged cluster gives us a cue. The graph you see on this slide is based on the results of the clustering algorithm, it is called a scree plot. The graph shows us how great the differences are that are bridged with each merger in the clustering algorithm. The distance that is bridged in the last merger is a lot larger than the difference that is bridged in the second last merger, and so on and so forth. In this scree plot, this would be one cluster, two clusters, three clusters, four clusters, and so on and so forth. This graph allows us to identify the ideal number of clusters. We want to be as low as possible in the graph with the lowest number of clusters that is possible. Ideally, we would like to be somewhere in the lower left corner, which would indicate that much variability is explained by a small number of clusters. In our case, a solution with four clusters constitutes a good compromise and the best approximation of being in this corner of the graph.

This leads us to the 4-cluster solution that you see here. If we are interested in the changes that the get-passive has undergone, then it makes sense to group these data points together and investigate what makes them differ from each other. We would find out what the early uses of the get-passive were like, what happens in this plateau here, and what happens in the later periods, in which many new examples with the construction appear.

To conclude this little clustering interlude, Variability-based Neighbor Clustering shows that in the case of the get-passive, the trend has four different temporal stages. The clustering algorithm gives us a periodization of the 


\section{4-cluster solution for the get-passive}

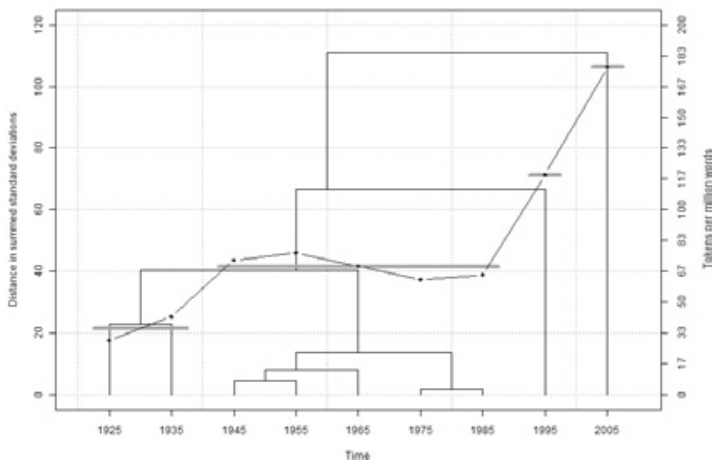

FIGURE 17

development. One thing that is unusual or at least not common in other types of periodization is that the periods are not of equal length. Normally, when linguists divide diachronic data into periods, they will choose equidistant periods such as centuries, half centuries, or blocks of 30 years. Here the periods can have different sizes. The clustering algorithm provides average values. In this case, the average values represent text frequencies. VNC can guide you towards structures that may otherwise go unnoticed or be hard to characterize objectively. I'd like to come back to the V-ment construction now. What does this method allow us to do with the changing productivity of the V-ment construction?

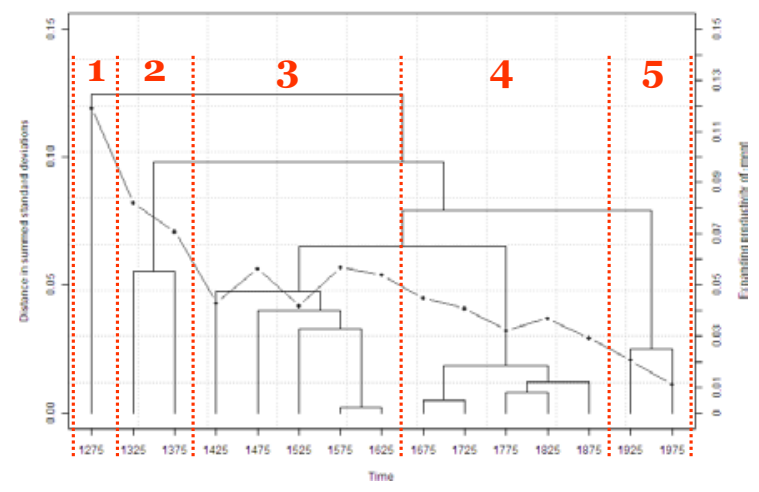

FIGURE 18 
This slide shows once more the declining curve of expanding productivity. I took this curve and ran the VNC algorithm over it. The algorithm went through these measurements, compared the differences from one period to the next, and merged those that were the most similar to each other. What you see here is the clustering solution that the VNC algorithm produces. I used a scree plot of the kind that you saw earlier to determine how many clusters I would be justified to assume in this development. The scree plot indicated a distinction between five clusters, which allows us to account for a fair amount of variability without assuming too many clusters.

Overall, I cut up the development into five different time slices that are not all of the same length. The first period is just one half-century. Then we have two half centuries, the third cluster covers a slightly longer period. The fourth period describes a long decline. In the fifth cluster we have the modern period with which the development ends.

In the remaining time that I have, I want to talk about how these different periods can be analyzed, and what we can actually learn about the construction and its development on the basis of this periodization. Using the VNC algorithm to find periods is interesting, but it is really just the preparation for the actual analysis. It is a way of cutting up the data, but what you do with the data is something else.

For the analysis itself, I decided to determine a range of relevant variables that pertain to form and function of the $V$-ment construction. I took all the 1400 types that I had in the database and annotated them for these variables. Then I used a multivariate statistical analysis to explore whether there were patterns of variation that would change over time. Let me start by talking about the different variables that matter to the construction.

\section{Variable 1: Etymological source}

Is a form borrowed or derived?

B: achievement, detachment, enforcement

D: bickerment, erasement, shipment

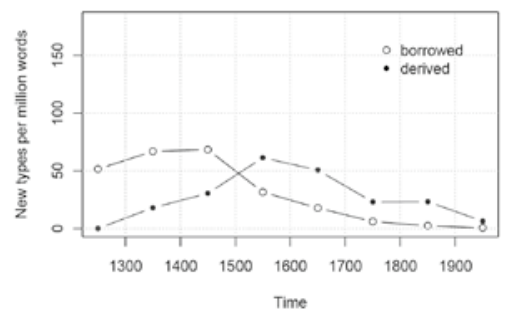

FIGURE 19 
The first variable is concerned with the etymological source of the construction. If we have a V-ment word, is that word borrowed from French, or is it native to English? Is it derived? There are borrowed forms like achievement or detachment or enforcement that have Romance stems. Then there are forms like bickerment or erasement or shipment that are Germanic in origin. You see in this graph that we have more borrowed types at first, but that they decrease from $145^{\circ}$ onward. The derived forms peak around 1550, and then they decrease too.

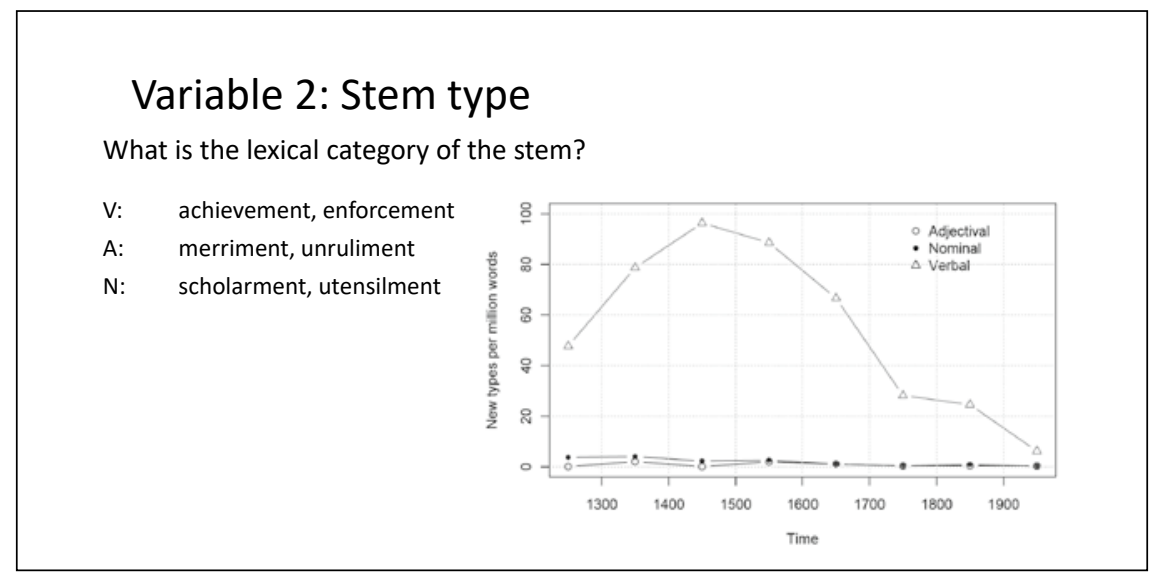

FIGURE 20

The second variable is concerned with the stem type. I mentioned that most types that we find have a verb. Achievement has achieve, enforcement has enforce, but there are types with adjectives, such as merriment and unruliment. There are types with nouns such as scholarment or utensilment. What you see in this graph is that these adjectival and nominal types are very rare, and that they occur only early in the history of the construction. Most types fall into the verbal category.

The third variable concerns the internal structure of the $V$-ment types. What is the internal hierarchical structure? There are words with a binary morphological structure. Judge is a monomorphemic word in English. Judgment is a word that has two morphemes. Treatment illustrates the same category. Then there are left-branching types, that is, words such as enrichment. The verb enrich is internally morphologically complex. The verbal stem of belittlement has two morphemes, and -ment attaches to it. Lastly, there are right-branching types. Those would be words such as ecomanagement, which is based on the word management that is prefixed by eco-. 


\section{Variable 3: Branching structure}

What is the internal hierarchical structure?

Binary: judgment, treatment

Left-branching: [en+rich]ment, [be+little]ment

Right-branching: eco[manage+ment], non[agree+ment]

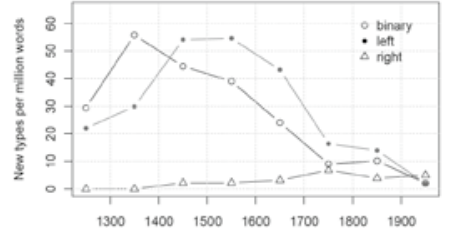

Time

FIGURE 21

Looking at the frequencies, we see that at first the simple binary branching types dominate. They are then superseded by the left-branching types. The right-branching types only come in very slowly and very gradually, but they continue to rise in frequency even through the very latest periods. That already prefigures that they are actually represent something different than the $V$-ment construction itself.

\section{Variable 4: Transitivity}

Does the form evoke an entity that is acted upon?

Transitive: $\quad$ arousement, punishment

Intransitive: flourishment, merriment

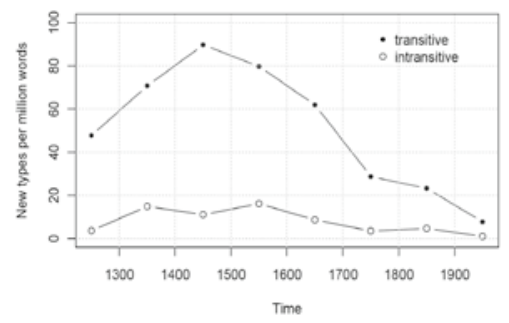

FIGURE 22 


\section{Variable 5: Semantic types}

Which overall meaning is conveyed by the form?

Action: confrontment, dismantlement

Result: settlement, scholarment

Means: ornament, refreshment

Place: parliament, environment

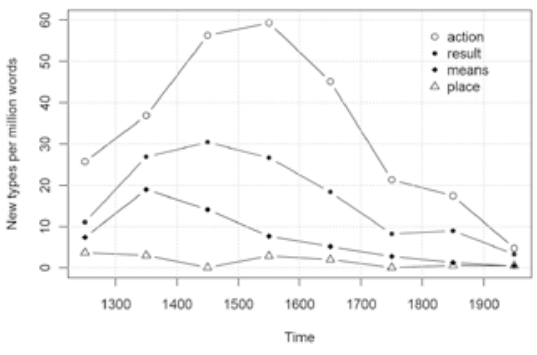

FIGURE 23

The next variable is concerned with meaning, and specifically with transitivity. For this variable, I relied on the argument structure of the verbs that form the stems of many of the $V$-ment types. Does the form evoke an entity that is acted upon? Verbs such as arouse or punish are transitive verbs that evoke a patient argument. By contrast, verbs such as flourish do not take a patient argument. They are intransitive verbs. This graph shows that there are many more transitive types than intransitive types. Both categories show decreases from around 1450.

Variable five is concerned with the different meanings that are conveyed by the $V$-ment construction. I already mentioned that the construction can express an action, as in confrontment, the action of confronting someone. It can also express results. A settlement is not the act of settling down, but the actual structure that characterizes a human dwelling place. There are types that express means. I have mentioned refreshment as an example. There is a fourth category that I assumed, namely, places like environment, for instance, or parliament. This category encompasses everything that I couldn't characterize as action, result or means.

All the variables that I have presented here are categorical. They represent choices between different categories, not continuous values that you can measure, such as frequency or degrees of productivity. Cross-tabulating categorical variables allows you to explore whether there are any interesting asymmetries in the way the data are distributed. 


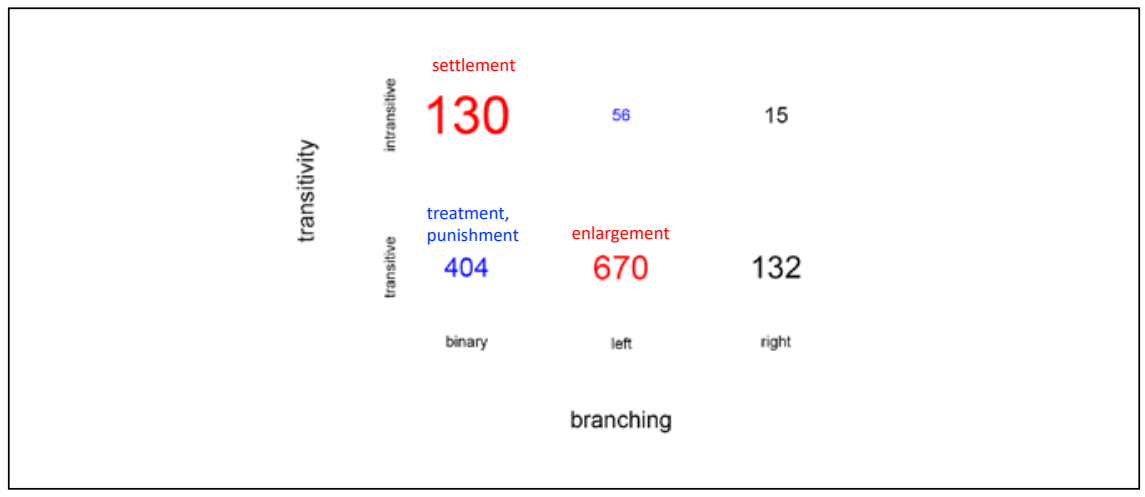

FIGURE 24

Here you see a cross tabulation of the transitivity variable with the variable of morphological branching structure. The rows show how many transitive types and intransitive types are in the database. The columns show how many binary-branching, left-branching and right-branching types are in the database. The observed frequencies that we have here can be compared against the expected frequencies that would be assumed if the distribution is random. A test such as the chi-squared test can determine whether the observed frequencies differ significantly from a random distribution. This kind of logic is visualized on the slide. If a number is shown in red, that means that the number of examples are significantly higher than expected. Blue numbers mean that a cell is significantly underrepresented. There are fewer examples than we would expect by chance. I have tried to visualize that also with the size of the font. We have 130 intransitive binary branching examples, and those are many more than we would have expected by chance. The other two intransitive types are relatively infrequent. To find that many in the upper left corner is something that is detected by the statistical test. You further see that in the upper row, most examples are in the leftmost cell. That is very different from the lower row, where left-branching transitive types, which are situated in the middle, are clearly overrepresented. Underrepresented forms include treatment or punishment, transitive, binary branching types. Overrepresented forms would be transitive, left-branching types like enlargement and also intransitive, binary-branching types like settlement.

Cross-tabulating two variables is just the start of the analysis. As you know, I didn't just have two variables. Of course, it is possible to cross-tabulate more variables than just two.

If you add a third variable, you have no longer a table that crosses two variables, but rather you have a cube that cross-tabulates three variables, but the 


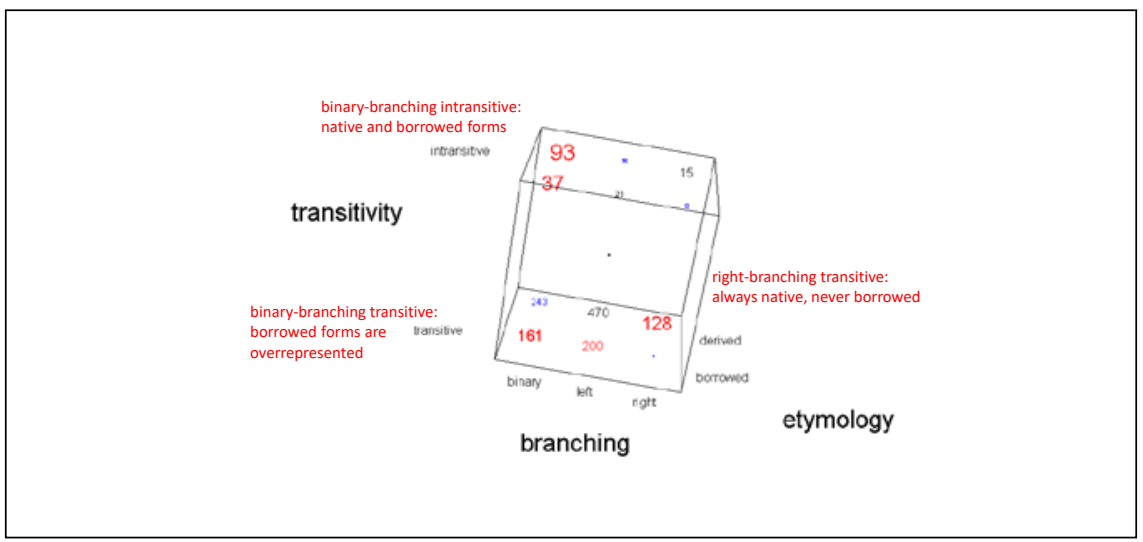

FIGURE 25

logic is essentially the same. For each cell in the cube you examine whether the types in the cell are overrepresented or underrepresented. Again, red numbers indicate that a cell is overrepresented, blue numbers indicate that a cell is underrepresented. Something that is clearly overrepresented in the data is the configuration of right-branching, transitive, and native types: forms like embodiment. Binary-branching transitive and borrowed forms like judgment are overrepresented as well. When we look at binary-branching intransitive types, both native and borrowed forms and are overrepresented. You can visualize this with up to three variables, but after that, ordinary physical space runs out of dimensions. Luckily, the computer can still handle it.

There is a method that is called configural frequency analysis which you can use for this purpose. The method is described in Stefan Gries' works (2009: 248). The method cross-tabulates a set of categorical variables and examines differences between observed and expected frequencies. I cross tabulated all 1400 types for the variables that I described earlier, taking into account the historical period, the etymological source, the stem type, the branching type, transitivity, and the semantic type. I wanted to find out whether there were configurations of values that would occur with greater than chance frequency during early periods of the data and later periods of the data. I wanted to see if early types would differ from later types.

In the remaining ten or so minutes that I have, I will go through the results very briefly. What happens during the early history of the $V$-ment construction? The first type, the first configuration of features that is overrepresented, is illustrated by forms like commencement, which are borrowed types that have a transitive verb stem. Types such as imprisonment, conferment, enchantment or judgement, those are words that are overrepresented early on in the history, 
and this is consonant with results that earlier studies have obtained. One consensus is that early -ment types typically had transitive verbs as hosts. That is confirmed by the data. Let me move on to the second period.

During the second period there are certain oddities that we do not find later. This period sees the emergence of types such as ointment, that is, a cream that you put on a wound to make it heal better. These are borrowed types that have a verbal stem. The stem is transitive, the forms are binary branching. What is crucial about these types is that they describe a means, so an ointment is a means to healing your wound. The semantic type of means is not very frequent overall, but it rises to a moderate level of frequency during this particular period, and that is what the analysis picks up.

There is another type that also describes a means. That type is illustrated by words like vesselment. These are special because they have a nominal stem. There are a few others, including monument, but not many. This is a highly infrequent type, but because it is more frequent than expected, it shows up here.

Moving on to the third period, here we finally observe what we can call the prototype of the construction, words like enlargement. These are types that are natively formed with a verbal stem that is transitive, that is left-branching and that expresses an action. We have not only enlargement, we have disbursement, misusement, renewment and so on and so forth, lots and lots of types. This in fact is the most frequent configuration in the database. There are 174 types in period 3 alone, but there are more than 300 in total. This is very much the conceptual and formal core of the construction. This I found is interesting because Plag (1999: 16) comments on this construction and observes that there are certain forms are acceptable even though they are unattested, they do not exist as words in English. Even though the construction is unproductive, if I use a form that conforms to this prototype, people will accept it as a legitimate word. This concerns unattested forms such as encodement and envisionment. Those are not real words, but they look close enough to the prototype that we were fooled into thinking that we may have heard this word at some point or other. That is in line with the present analysis. Neologisms may be fine if the host is a prefixed transitive verb.

During this time, another configuration is overrepresented. There is this strange but short-lived fashion of words like merriment, which have an adjectival stem. This is only found in natively derived words, not in borrowed words. It is very innovative, but it is really short-lived. All types like merriment or coldment or jolliment or adjustment occur in a very short time span of about 60 years. 
We are almost at the end. During this period that marks the decline of the construction, we find the overrepresentation of right-branching types, like disembodiment. We have something like embodiment and prefix it with dis-. That means it is not really the productivity of -ment that is at stake. Rather, the existing types of -ment are cannibalized upon by other prefixes. New forms are coined on the basis of existing types, which yields maltreatment, overenrichment, reemplacement, and so on and so forth. These are typically coined on the basis of the prototype form, like enlargement. This is thus an outgrowth of the prototype that is independent of the productivity of the suffix itself. This explains why the right-branching types actually continue to grow, even when everything else is in decline.

This continues through the last period, which gives us another overrepresented type that is also right-branching, but that does not verbalize an action, but rather a result. Malnourishment is the result of not having eaten enough for a long time. This type again dodges the strong trend towards the meaning of an action and it represents a metonymic shift from actions to results.

To come to an end, I started with these four questions and I briefly want to summarize the answers. The $V$-ment construction is a combination of a lexical stem and the suffix associated with different meanings. For our purposes, we can think of it as a network of constructions that grows and then fades over time. There are different patterns, different sub-patterns, like the type that leads to the form merriment. It is an outgrowth, a little part of the network that flourishes at some point and then contracts again. We have the prototype that is at the center of the network that starts as a cluster of borrowed forms and then expands on the basis of natively formed words.

I further asked how the construction changed in productivity. I said that contrary to proposals that were made earlier, there is really just one peak in productivity, which coincides with the nativization of the construction. My overall interpretation of that was that the construction was successful at first, but then faded very quickly. With regard to changes in form and function, the changes that we can mark here are the construction became nativized and a prototype emerged.

In the following, sub-constructions come and go. Lastly, the construction itself dies, and its types contribute to other developments, such as the emergence of right-branching types. Ultimately, productivity comes to a complete halt.

What about the puzzle that I started out with, i.e. lots of hapaxes but no productivity? My conclusion is that the remaining types and the right-branching words that are formed on the basis of it give us a sense of productivity that is 
actually misleading, a kind of false productivity that inflates our expectations of how productive -ment really might be, if we are just looking at the suffixes and do not consider the rest of the structure.

What about constructional change? The changes that we observe here do not really fit the mold of common grammaticalization processes that I have been talking about them in earlier lectures. If we are looking for processes such as bleaching or host-class expansion or erosion or decategorialization, we do not really see that. The $V$-ment construction does not undergo grammaticalization. It also does not undergo lexicalization. We observe something else. The $V$-ment construction is created as speakers generalize over lexical borrowings for a brief period, then speakers experiment with this generalization and after a while the construction ceases to exist as a cognitive schema and just its lexical instantiations remain.

For the changes that we see here, we could decompose them into general processes of language change, like semantic change, reanalysis perhaps, but that would fail to explain a number of phenomena that I actually see as crucial to the history of the construction. The emergence of a derivational schema from borrowed forms, prototype effects like Plag's observation that a form such as envisionment is acceptable, and then the fading fashions of merriment and other forms like that. This means that the generalizations that we see in the history of the $V$-ment construction are local. We are looking at a network of constructions and sub-constructions, and these generalizations will sometimes capture the data more adequately than the broadest possible generalization. I mentioned the cognitive commitment that was proposed by George Lakoff earlier in this lecture series. Lakoff proposed another commitment, namely the generalization commitment. You try to find the broadest generalization wherever this is possible. I agree that the job of scientists is to find generalizations. We are supposed to connect the dots and state our insights in the broadest possible terms. However, sometimes local generalizations are really what is more crucial. The broader generalization might be an elegant theory, but it might not be an accurate reflection of what goes on in speakers' minds. When Ewa Dąbrowska spoke at this forum (2017), she made this point very eloquently, so I will just leave it at that here. The bottom line is that the analysis of language change can profit from adopting a constructional point of view that takes seriously this idea of constructional networks. With that I would like to come to an end and thank you once again for your attention. 